\title{
Analyzing, Modeling and Predicting Organizational Effects in a Distribuited Sensor Network*
}

\author{
Bryan Horling \& Victor Lesser
}

Multi-Agent Systems Lab

University of Massachusetts, Amherst, MA

\{bhorling, lesser\}@cs.umass.edu

\section{ABSTRACT}

The organizational design of a distributed system defines how entities act and interact to achieve local and global objectives. We describe how a system employing different types of organizational techniques has been used to address the challenges posed by a distributed sensor network environment. The high-level, multi-agent architecture of this realworld system is given in detail, and we provide empirical results demonstrating the effects the organization has on the system's performance across several different metrics. As with any design, the particular approach that is employed makes trade-offs, some of which are obvious and some more subtle. The presence of such trade-offs motivates the need for a better understanding of precisely how the organization influences large and small-scale behaviors. To address this need, we first demonstrate how a collection of analytic models can be developed to predict such effects. This experience is then used to ground the presentation of a more comprehensive, domain-independent organizational modeling language called ODML. The structure and capabilities of ODML are explained through the construction of a unified model of our sensor network organization. We then show that this model provides an accurate prediction of the original empirical results.

*This material is based upon work supported in part by the National Science Foundation Engineering Research Centers Program under NSF Award No. EEC-0313747. Any opinions, findings, conclusions or recommendations expressed in this material are those of the author(s) and do not necessarily reflect the views of the National Science Foundation. Effort also sponsored in part by the Defense Advanced Research Projects Agency (DARPA) and Air Force Research Laboratory Air Force Materiel Command, USAF, under agreement number F30602-99-2-0525. The U.S. Government is authorized to reproduce and distribute reprints for Governmental purposes notwithstanding any copyright annotation thereon. The views and conclusions contained herein are those of the authors and should not be interpreted as necessarily representing the official policies or endorsements, either expressed or implied, of the Defense Advanced Research Projects Agency (DARPA), Air Force Research Laboratory or the U.S. Government.

\section{INTRODUCTION}

Distributed vehicle monitoring as an example application of distributed situation assessment and more generally distributed resource allocation has been studied in the multiagent systems community since its infancy [21,13]. This environment is particularly interesting when investigating issues of scale, because practical scenarios can be envisioned employing distributed sensor networks that are arbitrarily large both in number and geographic size, making purely centralized control inefficient. Each network member would have some type of data producing or interpretation capabilities, resulting in a potentially overwhelming amount of information requiring analysis. Shared resources, potentially conflicting goals and the need to adapt sensing policies in real time to emerging phenomena add further complications. These challenges make it an ideal candidate for multi-agent techniques.

Our solution, which we will describe in detail in Section 2, uses organizational structures as a key component to address these problems. Rather than employing a single organizational scheme, we have found that exploiting the strengths of a collection of different organizational styles can be quite effective. Our choice was based on our experiences working with a large-scale, realistic distributed sensor network over the past four years, both in detailed simulations and on real hardware [14].

The organizational design used in our solution is intended to address the challenges that arise through scale, by exploiting locality of reference and organizational constraints to impose limits on how far classes of both control and data messages propagate. The environment's most limiting resource is the wireless communication medium, and we will therefore use this resource throughout the paper to describe the effects of the organization. Our design uses environmental partitioning to create localized regions of interaction, called sectors. Within these sectors, agents take on different responsibilities that dictate their individual behaviors. A consequence of this approach is that the number of sensors in these sectors affects how efficient the system is, since large regions may create unwelcome disparities in communicative or processor load, and small regions cause a more global increase in overhead. Specifically, we will see how sector size affects the overall communication load, load disparity between agents, average communication distance, and the quality of tracking. By varying just this one aspect of the organization, we will show that the performance of the system can be greatly influenced by the organization's design parameters.

The notion of "organizational design" is used in many different fields, and generally refers to how members of a society act and relate with one another. This is also true of multi-agent systems, where the organizational design of a system can include a description of what types of agents exist in the environment, what roles they take on, and how they interact with one another. The objectives of a particular design will depend on the desired solution characteristics, so for different problems one might specify organizations which aim toward scalability, reliability, speed, or efficiency, among other things. To date, relatively little work has been done in the multi-agent community analyzing the characteristics and tradeoffs of different organizational types.

Complicating the design process is the fact that many potentially important characteristics can be subtle, and not readily identified as the system is being developed. For example, as alluded to above, certain global characteristics improve as we vary the sensor network organization, while other local characteristics degrade. The underlying mechanisms causing this can be complex and interdependent, making it difficult to create the correct design for a particular working environment.

It is our belief that understanding the root causes of these characteristics and developing accurate quantitative models of their effects are both critical to selecting an appropriate design, particularly as the agent population grows in scale or complexity. Once derived, this same knowledge can also be put to good use in verifying and changing the organization at runtime in response to changing conditions, creating a more robust and adaptive system. We will demonstrate how analytic models of our organization can be devised to help obtain this understanding. We will then build upon these ad hoc models by introducing a new language designed to capture organizational information in a single unified, predictive structure. Such models can help answer the questions that we have posed, by using quantitative knowledge to represent interdependencies, predict performance, and allow subtle effects to become more transparent.

The remainder of the paper is divided into four main sections. In Section 2, we will describe the sensor network domain and our organization-based solution in more detail. Following this, we will describe a series of tests that were performed to evaluate the effect the organization has on the system's performance across a range of metrics. In Section 4 , we will show how these characteristics can be quantitatively modeled with a set of equations. Finally, in Section 5, we will introduce ODML, a domain-independent organizational modeling language. We will use ODML to create a unified model of the sensor network organization, and show how it can be used to predict both large and small-scale organizational effects. 


\section{DOMAIN AND ORGANIZATION OVERVIEW}

The goal of a distributed sensor network is most generally to employ a population of sensors to obtain information about an environment. In this paper, we will focus on using such a network to track one or more targets that move along arbitrary paths in an area. A collection of threehead, MTI Doppler radars make up this network [14]. They are each fixed in position and have a wired power source. Each sensor is equipped with a processor, on which is run a single process that controls the sensor. We will call this local process an agent. The sensors are connected with a FM-based wireless network, which is divided into eight communication channels. Each channel has limited capacity, and agents may communicate over only one channel at a time.

Individual sensors can return only simple amplitude and frequency values, so a sensor is incapable of determining the absolute position of a target by itself. In addition, because only one of a sensor's three heads may be in use at a time, each sensor's scanning policy must be adapted based on current needs. To track under these conditions, the sensors must be organized and coordinated in a manner that permits their measurements to be used for triangulation, and geographically distinct groups of such coordinated sensors used to produce a continuous track as the target moves. More measurements, and particularly more measurements taken in groups in the same area at approximately the same time, will lead to better triangulation and a higher resolution track. To accomplish this, our architecture employs closed-loop control; the measurements and estimated target locations are used by the sensor agents to evaluate and adapt the network's subsequent scanning strategies. Consequently, any processing, decisions making and communication that occurs to enact this control has to take place in real time, or the target may be lost. Additional hurdles include a lack of reliable communication, the need to scale to hundreds or thousands of sensor platforms over a wide area, and an uncertain, noisy operating environment. The architecture, implemented in roughly 40,000 lines of Java code, has been demonstrated successfully in both simulation and real-world experiments. A more detailed description of the entire framework and the environment it operates in can be found in [14].

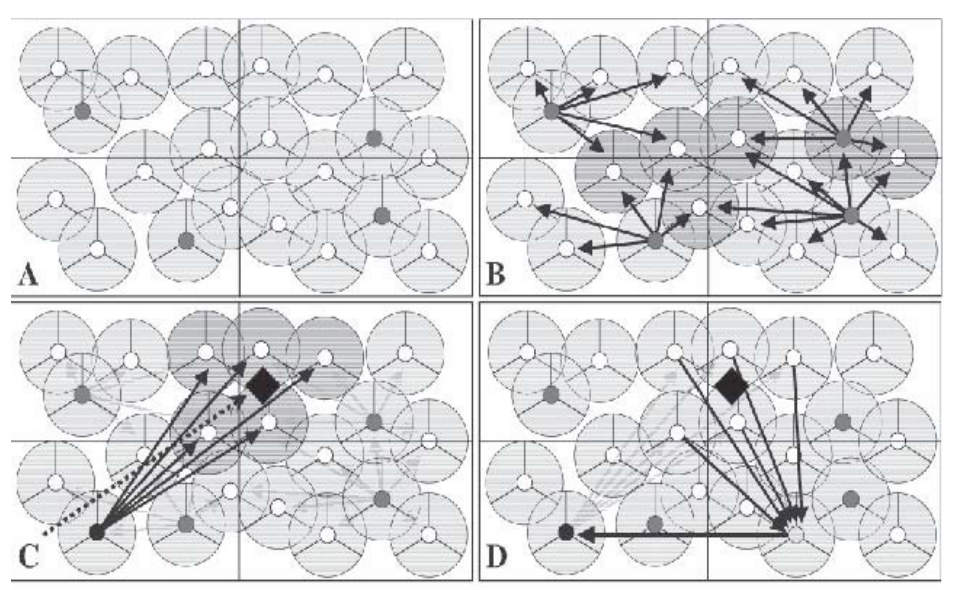

Figure 1. High-level architecture. A: sectorization of the environment, B: distribution of the scan schedule, C: negotiation over tracking measurements, D: tracking data fusion.

As mentioned above, we have employed an explicit organizational design in an effort to reduce overhead without negatively impacting performance. There are three types of responsibilities, or roles, that agents may take on: sector manager, track manager and sensor manager. Sector managers are created for each sector in the environment, and serve as intermediaries for much of the local activity. For example, they generate and distribute plans needed to scan for new targets, store and provide local sensor information as part of a directory service, and assign track managers. Each detected target has such a track manager, which is responsible for identifying the sensors needed to gather target information, gathering the resulting data, and fusing it into a continuous track. Track managers obtain some information from their originating sector manager, but can also interact directly with other sector and track managers. The sensor manager role controls how the local sensor is used. In response to sector or track manager requests, it takes measurements at specified times and places, and reports back the resulting data. Each of these three responsibilities corresponds to a role in the organization, which must be assigned to a particular agent. Agents may work concurrently on one or more of these roles, so a viable organizational design must ensure that each agent has sufficient resources to meet the combined demands of the roles it is assigned.

As we will show, some aspects of this design are static, such as the partitioning and sector manager assignment, and defined as the sensors are deployed in the environment. Other aspects are dynamic, such as the track manager assignment and sensor selection, requiring the agents to self-organize in response to new events. This blend of styles takes advantage of characteristics of the environment that are invariant, without giving up the ability to react appropriately as conditions change.

To see how the organizationworks in practice, consider the scenario in Figure 1. The environment is first divided by the agents into a series of sectors, each a nonoverlapping, identically sized, rectangular portion of the available area as shown in Figure 1A. In other work we have also explored the use of heterogeneously-sized sectors [20]. The intent of these divisions is to limit the interactions needed between sensors, to reduce and distribute the overall communication load. As we will show in Section 3 , this strategy does not always have the desired effect.

Each sensor has a local agent that takes on a sensor manager role. A single agent in each sector also takes on the sector manager role, represented by shaded inner circles in Figure 1A. Sensor managers begin their existence by finding their local sector manager, and sending it a description of the sensor's capabilities. These include the sensor's position, range, orientation and preferred communication channel. When completed, the sector manager will possess a complete picture of the sensing capabilities within its sector, which it offers to other agents in the form of a directory service. The sector manager also uses this information to generate a scanning schedule for detecting new targets, which it disseminates to the local sensors in Figure $1 \mathrm{~B}$.

Once the scan is in progress, individual sensors report positive detection measurements to their sector manager. The sector manager, through interactions with nearby track managers, maintains a list of targets currently close to or within its sector. By comparing the measurement with that target list, the sector manager can determine if a new target was found, or if it is more likely the measurement was of an existing target. If it determines a new target was found, the manager selects an agent from its sector to be the track manager for that target. Not all agents are equally qualified for this role, and an uninformed choice can lead to very poor tracking behavior if the selected agent is already busy or shares communication bandwidth with garrulous agents. For example, if we simply collocated the track manager and sector manager roles at the same agent, the combined communication load will generally exceed capacity. Conversely, if an agent who has previously acted as a track manager is chosen, some of the environmental state that agent had accumulated may be reused, which reduces its communication needs. Therefore, in making this selection, the sector manager considers each of its agents' estimated load, communication channel assignment, geographic location and history. Recognizing such ramifications of role assignment will be an important aspect of the analysis we present in 
Section 5.

The track manager role, depicted in Figure $1 \mathrm{C}$ with a blackened inner circle, is responsible for tracking the target assigned to it. To do this, it first discovers sensors capable of detecting the target, and then negotiates with members of that group to gather the necessary data. Discovery is done using the directory service provided by the sector managers. As the target approaches a previously unknown area, the track manager will query the appropriate sector manager to determine the available local sensing capabilities. The track manager uses this knowledge to determine from where and when the data should be collected, and sends measurement requests to the sensor managers it selects (see Figure 1C). Because those sensors may be servicing tasks from other sector or track managers, conflicts can arise between the new task and previously existing commitments. The sensor agent will address such conflicts as best it can locally by using priorities to de vise a round-robin schedule, but will also notify the conflicting managers of the problem. Because these managers have a more global view of the situation, they are in a more suitable position to resolve it. For example, they may negotiate to use other sensing resources, or offer concessions in the form of reduced quality. This process is described in detail in [15].

The data produced by the sensors is collected and analyzed (see Figure 1D). Although this activity is logically a separate role, it is a relatively lightweight process, and as a simplification our organizational design implicitly incorporates it into the track manager's responsibilities. Once the track manager has received the measurements, the data are fused in a triangulation process. Amplitude and frequency values can place the target's location and heading relative to their source sensor, and several of these relative values can be combined to derive an absolute position. The data point is then added to the track, which is used to predict the target's future location. It is also used to periodically notify nearby sector managers of the target's location.

At this point the track manager must again decide which sensors are needed and where they should take measurements. Under most situations, the process described above is simply repeated. However, if the target has moved far from where the track manager is, the track managing role may be migrated to a new agent in a different sector. This is done to avoid penalties associated with long-distance wireless communication, which may cause unwanted latency or unreliability transferring information. This technique is covered in more detail in Section 3.3.

\section{EMPIRICAL EVALUATION}

The two primary organizational features used by this system can be thought of as geographic coalitions and functional differentiation. The first describes the partitioning process, while the second is a result of the heterogeneous assignment of roles to agents. An integral part of each is the notion of locality. Information propagates and is made available to only the agents which have need of it. In some cases, such as with the environmental sectorization, artificial boundaries are created to encourage locality at the expense of time or flexibility. In other cases, as with the target tracking role, locality is exhibited naturally through the domain.

There are many data flows and interactions that are encouraged and restricted by this design. As we will demonstrate, these characteristics affect the quantitative performance of both individuals and the system as a whole in a variety of ways. We will informally describe these effects below, and provide more concrete descriptions in Sections 4 and 5.

In the following sections, we will show empirical evidence exhibiting these characteristics, and explain how they drive the selection of an organizational design. Our experimental scenario consisted of a group of 36 sensors and 4 mobile targets. Different sized sectors were tested in this scenario, ranging from 36 very small sectors each containing just one sensor, to a single sector encompassing the entire area. All sectors shared the same size within any given test. Our initial hypothesis was a reasonable sector in our environment would contain from 6 to 10 sensors. We will show later that "reasonable" in this sense depends on a number of factors, including the density of the sensors, sensor range, communication medium characteristics and target speed, among other things. The sensors were arranged in a grid pattern and the targets' locations and movement spread evenly through the environment to normalize results and simplify analysis. Targets moved with constant speed. The results were then observed over 10 runs per configuration in a simulation environment called Radsim, which closely models the performance of the physical MTI sensors [11]. The same agent code was used for both simulation runs and actual hardware tests. Each run lasted approximately 140 seconds.

\subsection{GEOGRAPHIC COALITIONS}

Our first evaluation metric is the total amount of communication that occurs in the system. Figure 2 shows that as the number of agents in each sector increases, and there are correspondingly fewer sectors overall, the amount of communication traffic decreases. Because each sector requires a certain amount of control messages, the total number of messages is reduced as the number of sectors decreases. A more detailed view of the effects this change has on messaging will be shown later in Figure 4 .

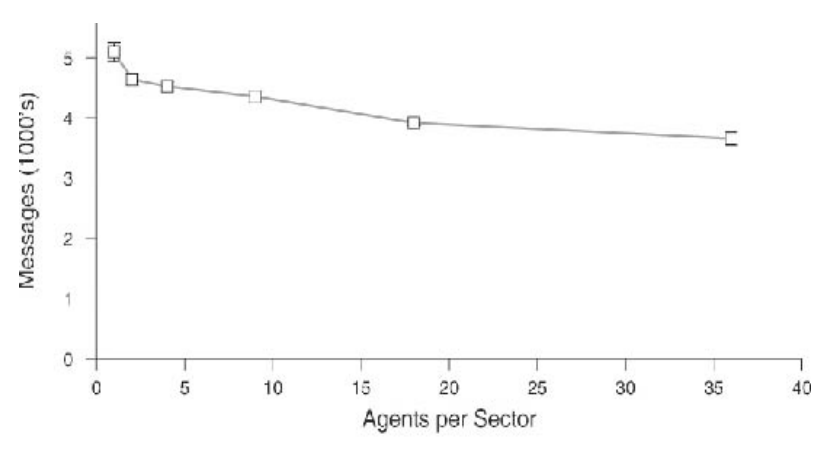

Figure 2 - Affect of sector size on messaging

Recall that our initial intent behind creating these sectors was to reduce the communication burden. The results in Figure 2 are in some sense contradictory of this goal, because they show that the unpartitioned environment had the lowest communication overhead. The partitioning process described in Section 2 results in the creation of loose coalitions of sensors based on geographic location. Sector directory information, new target scan schedules, discovery measurements and certain tracking control messages are all contained within or directed to these coalitions. Because the manner in which this information being communicated is determined by the sectors, the sectors' average size and shape has a tangible effect on some aspects of the system's performance. If the sector is too large, and contains many sensors, then the communication channel used by the sector manager may become saturated. If the sector is too small, then track managers may expend a great deal of time and bandwidth updating sector managers as its target moves through the environment. So, although these result show that large sectors have lower total overhead, the individual picture is not so straightforward. This is covered in more detail below.

Although not shown in this figure, partitioning can also affect reactivity, because time may need to be expended to discover sector information. A track manager, for example, must perform queries to obtain sensor information as its target moves to new sectors. Smaller, more numerous sectors will result in delays caused by the additional queries, which ultimately affects the number of measurements it receives. This delay will be revisited in Section 4 . 


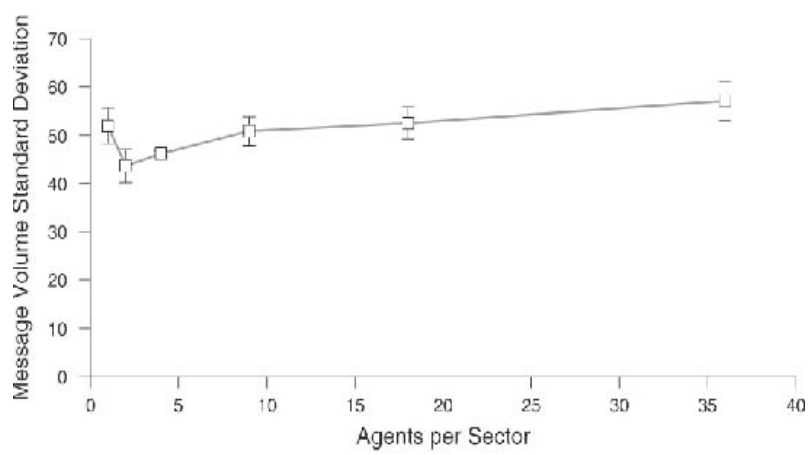

Figure 3 - Messaging disparity vs. sector size

\subsection{FUNCTIONAL DIFFERENTIATION}

The varied assignment of roles forms a different, functional organization [5] in the system. Agents specialize their functionality in order to restrict the type of interactions which must take place between agents. For example, to obtain information about available sensors, a track manager must only contact the relevant sector managers, instead of blindly broadcasting to all sensors [22]. Concentrating the track management functionality into individual agents serves a similar role, by limiting the number of interactions necessary to resolve conflicts in sensor usage.

Although this type of functional decomposition does reduce the total number of interactions an agent might need to make, it can also increase that number for particular individuals in the environment. For example, we have seen how the sector manager is responsible for disbursing information about the sensors in its sector, which facilitates the track manager's discovery process. However, by serving in this capacity, it also makes itself a center of attention, which can result in unreasonable load when demand is high.

Consider Figure 3, which shows how much agents in the population differ from one another in their communication habits, as the sector size changes. This notion is captured by measuring the standard deviation in communication activity (total messages sent) exhibited by individual agents. If all agents are roughly the same they will have a low deviation, while a population that has a handful of outlier agents with significantly higher message traffic will have a high deviation. As the number of agents in each sector increases, this graph shows an increase in disparity, because a few agents are communicating more than their peers. Since the environment and target spacing are uniform, the differences can be attributed to the roles those agents take on. The rise in deviation when there is a single agent per sector represents the coexistence of the sector and track manager roles, because all agents act as sector managers when there is only one agent in each sector. This trend demonstrates that as the sector size grows, specialized agents such as sector and track managers can become "hotspots" of activity. In a bounded environment with unreliable communication this concentration of activity could lead to reduced performance and data loss if the communication channel becomes overloaded.

A growing tension between sector sizes is made apparent by these results: Figure 2 shows that smaller sectors lead to increased message traffic, and while Figure 3 shows that larger sectors imbalance load in the population. Although not shown, similar trends were observed in agents' local workload levels, which track the number of non-communicative actions being performed. Both characteristics are bad, so a compromise must be sought between them in the selected organizational design.

\subsection{ORGANIZATIONAL MAINTENANCE}

As insinuated above, there are costs associated with creating and maintaining the organizations employed by this design. The most frequently updated aspect of the organization is the relationship formed between track and sector managers, because the sectors interacted with by the track manager change as the target moves. This results in a class of control messages dependent on sector size. For example, as the target moves into part of the environment the track manager is not familiar with, the manager must query the sector manager of that area to discover local sensors. Once those sensors are known, data collection commitments can be established.

As the target is tracked, the nearby sector managers must also be notified of the target's estimated position.

Figure 4 provides a quantitative view of this overhead. As sector size increases, fewer directory and tracking control messages are necessary, because there are a fewer sectors to interact with as the target moves. In addition, the number of measurements increases as the sector size increases, because the reduced time spent by the track manager interacting with the additional sector managers allows more time to be spent requesting data. More measurements results in a lower root-mean-squared (RMS) error between the measured and actual track, as seen in Figure 5.

The technique of migrating the tracking responsibility through the agent population as the target moves is another example of how locality can be exploited. Signal attenuation conspires to make communication less reliable as distance increases. Multi-hop protocols can maintain reliability, but will increase end-to-end latency at each hop. Lacking the capacity for movement, the initial manager selected to track a target will therefore become less effective as the target moves away from it. By migrating this task to follow the target, the organization is able to retain locality despite the fact that the sensors themselves are immobile. This results in a reduction in the average distance that messages must travel.

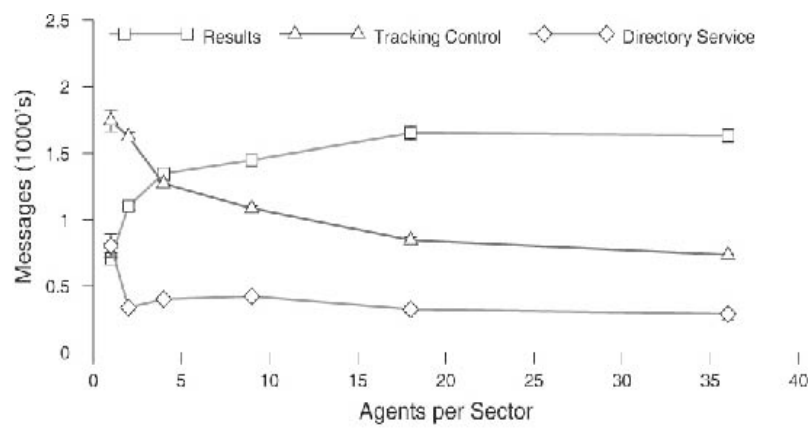

Figure 4 - Message types vs. sector size 


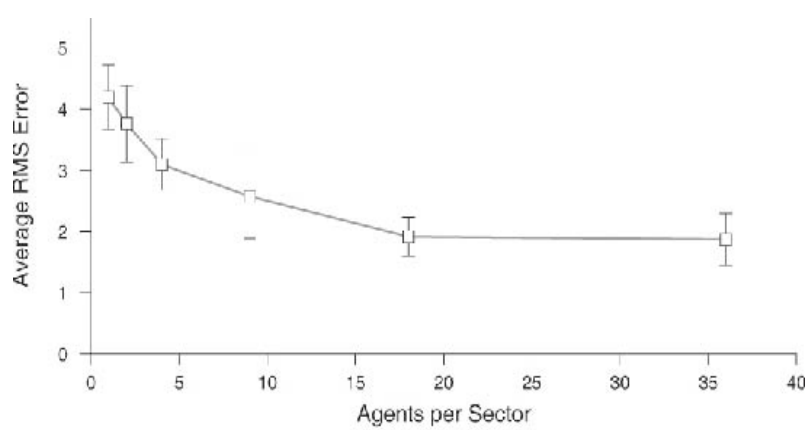

Figure 5 - Effects of sector size on RMS error

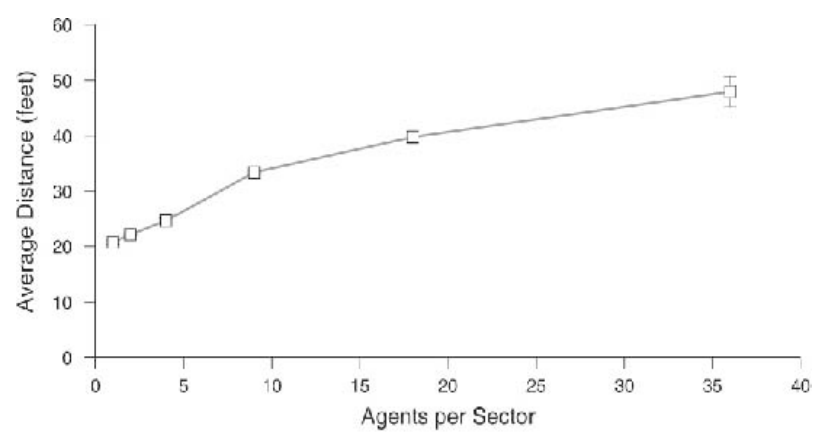

Figure 6 - Average communication distance

Figure 6 shows the effect track manager migration has on the average distance of communication. Because migration is triggered by sector boundaries, the tracking task will migrate less frequently when sectors are large, simply because they cover more area. Conversely, a lower average communication distance is observed when sectors are smaller. The lower migration rates also contribute to the increased measurement totals from Figure 4. Each migration interrupts the collection process as the role is moved from one agent to another, so the more frequently this transfer takes place, the more the average overall collection rate will be reduced.

These metrics contribute to the organizational tension. Large sectors improve the system's RMS error rate, while smaller sectors exhibit better communication locality.

\subsection{SCALABILITY RESULTS}

To explore the generality of these trends, we performed na additional set of experiments that varied numbers of targets. Each test contained between 1 and 24 equally distributed targets, all of which moved concurrently through the environment for the duration of the experiment. The scenario was otherwise identical to those in Section 3 . Figure 7 shows that our original communication disparity profile from Figure 3 is maintained as the target density is varied, and the amount of disparity increases with the number of targets. Intuitively, this is because the amount of work particular agents are performing is tied to the number of targets in the environment. The communication load of the sector managers, for example, is directly proportional to the number of track managers it must interact with. This is particularly true as the sector size increases - in the most extreme case a single sector manager must support all 24 track managers.

Similarly consistent results are seen for the systems RMS error, in Figure 8. The RMS error profile is maintained, although the baseline RMS error increases because the bounded sensing capabilities result in fewer average measurements per target. Notice how the RMS value for 6 and fewer targets are clustered together, while those with 8 or more become progressively worse. This is caused by a phase transition that occurs between 6 and 8 targets, when the number available sensors is no longer sufficient to meet demand. The inevitable reduction in the number of measurements track managers receive leads to an increase in RMS error.

Additional tests were performed which also varied the number of sensors in the environment, using six different configuration with between 9 and 81 sensors [7]. Results from those experiments concur with the trends outlined above.

The conclusion we draw from these experiments is that a tradeoff exists between communication volume and its distribution over the agent population. Message volume decreases when there are more agents per sector because fewer interactions are needed to obtain information, as shown in Figure 2 . However, this shift can cause individual agents to incur a disproportionate communication burden, as shown in Figures 3 and 7. Figures 4, 5, and 6 show that organizational maintenance causes a similar tradeoff - larger sectors have lower overhead and better RMS error, while more track migration in smaller sectors increases communication reliability. 


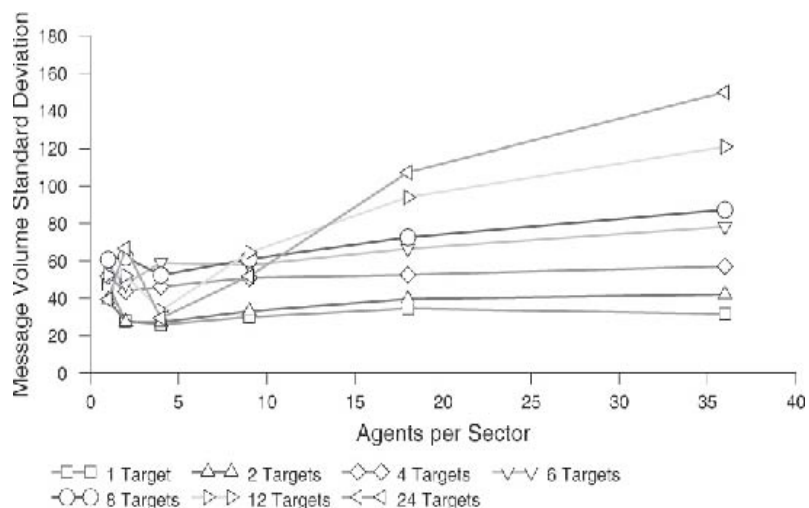

Figure 7 - Communication disparity with varied sector sizes and target densities

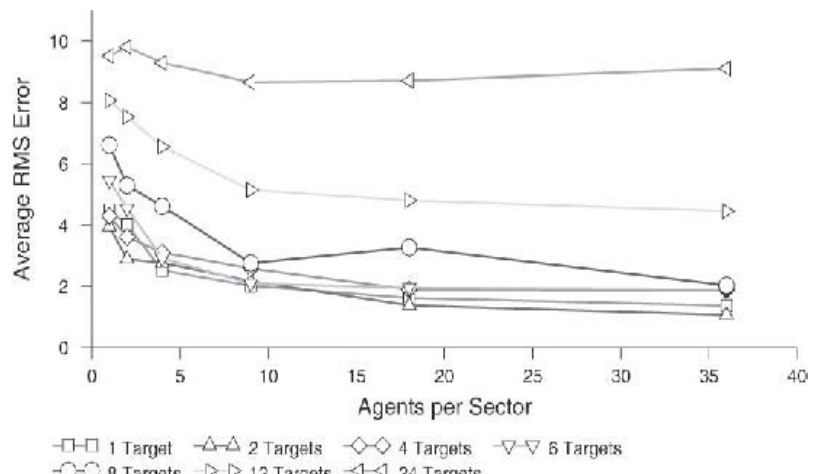

$-O 8$ Targets $\gg 12$ Targets 424 Targets

Figure 8 - RMS error differences with varied sector sizes and target densities

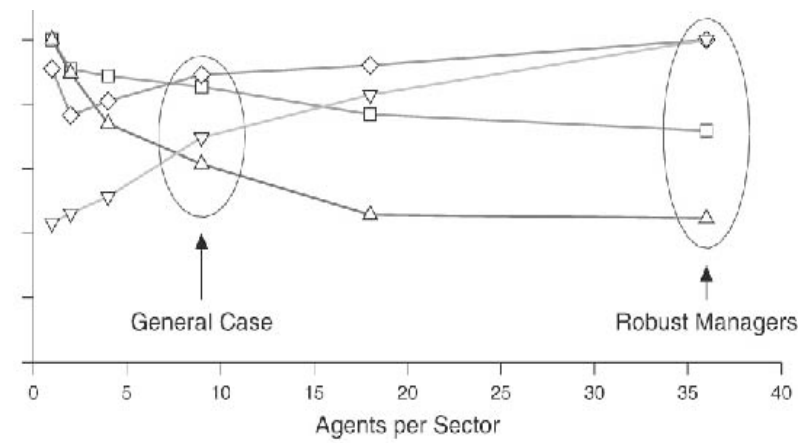

$\neg-\square$ Messages $\Delta-\Delta$ AMS Error $\diamond \diamond$ Comm. Deviation $\nabla \nabla$ Comm. Distance

Figure 9 - Finding the appropriate configuration from normalized results

\section{DISCRETE ANALYTIC MODELS}

Our long-term objective is to use results such as these to make architectural design decisions. A simple strategy might compare the metrics graphically, and select a point which seems appropriate for the expected conditions. Normalizing and overlapping the trends from Section 3, produces the graph in Figure 9 . By searching for a common inflection point in this diagram, we can conclude that a sector size between 4 and 9 strikes an acceptable balance between the competing positive and negative characteristics. This supports our hypothesis that a sector size between 6 and 10 was a reasonable choice. However, the notion of "reasonable" is problemspecific, depending on the characteristics of the agents, the resources they use, and the environment. For instance, if more robust managers were available to handle the increased load, this graph also shows that better RMS performance can be obtained by using larger sector sizes. In general, the requirements imposed by goals and the capabilities of the system and environment guide an appropriate selection, and these experiments only suggest a course of action for a particular configuration.

The use of a more formal, analytic model that incorporates the various characteristics can evaluate a wider range of candidate designs. Instead deducing metrics from a graph as above, one can create a function that takes requirements and characteristics as inputs, and produces a prediction or rating as output. To do this, one must capture the system's behaviors in an abstract, quantitative model that provides a good approximation of the real system. We will do so by predicting role-specific values over the lifetime $t$ of the role. As before, we assume that the sensors and targets are uniformly distributed in the environment, and targets move with constant velocity. One could relax these assumptions by estimating interaction probabilities; although the calculations would be more complex, the spirit of the analysis would remain the same. Similarly, one could determine worst-case 
peak performance by assuming worstcase densities. The formulas presented below do not represent actual message totals, but are meant to reflect relative growth rates. As we will show in Figure 10, quantitative results can be obtained through the addition of appropriate constants. Consider the sensor manager. We will define the number of measurement messages sent by the sensor manager as its measurement load ( ). Measurements are taken in response to track manager requests, which are in turn prompted by targets in range of the sensor. is therefore dependent on the likelihood that a target is within its range Assume $\mathrm{T}$ targets in an environment of area $\mathrm{A}$, each with $\mathrm{m}$ measurements per time unit. can then be approximated with the following equation:

(1)

$$
\mathcal{M}=\sum_{t} \min \left(\frac{\pi r^{2}}{A} T m, m\right)
$$

So, as the number of targets increase, or the environments area decreases, the number of measurements will approach tm. This model is an upper bound, however, as it does not take into account track managers' specific behaviors, such as delays or inefficiencies that could affect the rate at which measurements are requested. These will be made more explicit in our model of the tracking process below.

The sector manager's load is dependent on both the size of the sector and the number of targets. As we have observed earlier, larger sectors mean more sensors must be registered, as well as an increased probability that a target will be in the area.

can be broken down into the one-time costs associated with sector creation, when the sensors send descriptions of themselves to their sector manager, and the continuing costs derived from targets moving though the sector:

$$
\mathcal{S}=\frac{S}{A} N+\sum_{t}\left(\frac{\hat{S}}{A} T u+\frac{v}{\sqrt{S}} \frac{S}{A} N\right)
$$

size of the sector's area, while is the effective size of the sector's area. S and are differentiated by what they represent. S is the strict bounding area of the sector we have been discussing thus far; membership in the sector is defined by containment within that area. is the potentially larger area over which measurements can be taken by sensors in that sector. If for example each sensor has a range of $r=20$, then will be the area bounded by $S$ unioned with a perimeter of width 20 surrounding $S$. Because it is this effective area that determines when a track manager provides the sector manager with target location updates, grows in proportion with. The second term in the summation represents the directory queries it must respond to as targets enter its sector, which depends on the velocity of the target $\mathrm{v}$ and the average distance the target must cross before it reaches a new sector. This latter term depends on the probability of target turns and the shape of the sector itself; we model it with a very coarse estimate of the average chord length in the sector $\sqrt{S}$

Ignoring the effects of uncertain measurements or faulty data fusion, and assuming a reasonable choice of sensors are requested, the RMS error of the tracking process is primarily dependent on the number of measurements that are received over the lifetime of the track. In the absence of hindering factors, the track will receive measurements at a uniform rate $\mathrm{m}$ from each of $\mathrm{c}$ sensors used (we assume $\mathrm{c}$ is sufficient for triangulation purposes). The actual rate of measurements will be less than this, affected by the number of sensors that are used and any delays incurred by overhead tasks. In particular, the collection of sector directory information, and task migration when the target has grown too distant can reduce the total number of measurements that are obtained. Competition for sensors by other targets can also reduce the measurement rate. 


$$
\begin{aligned}
\hat{c} & =\min \left(c, \frac{\pi(b+r)^{2}}{A} N\right) \\
l & =\min \left(0, v\left(\frac{N}{\hat{c} T}-c\right)\right) \\
s & =\min \left(1, \frac{N}{\hat{c} T}\right) \lambda^{l} \\
i & =1-\frac{v}{\sqrt{S}}\left(d+\frac{g}{2}\right) \\
\mathcal{R} & =\sum_{t} \hat{c} m(s i)
\end{aligned}
$$

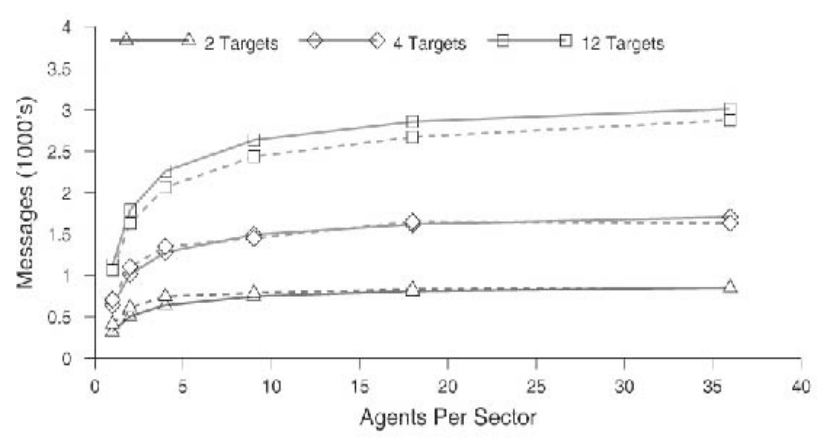

Figure 10 - Comparison of predicted and actual results of $\mathcal{R}$ for 2,4 and 12 targets. Solid lines are predicted, dashed are actual

Equation 3 defines, the number of sensors that will actually be used to track the target. It is bounded above by the desired quantity c, and below by the expected proportion of the total number of sensors that are in range of the target with radius $b$. This captures the effect that sensor density has on track quality. $s$ models the proportion of a potentially contended sensor's time usable by the target. If we assume the sensor is shared equally among targets, then the measurement rate obtained by an target will be inversely proportional to target density. As sensors come under contention, an allocation strategy must be employed to resolve the conflict [15]. An additional reducing factor models this optimization process; I estimates the amount of conflict, while $\lambda$ controls how much the conflict degrades performance. When the target moves into a new area, there will be a delay $\mathrm{d}$ before the appropriate information is received. An additional delay $\mathrm{g}$ is incurred during track migrations when the target has moved two sectors away from that of the track manager. The net effect $i$ of these delays and the corresponding increase in measurements when sector sizes grow is supported by Figures 4 and 5 .

To evaluate the accuracy of track measurement model, values were determined for each of the variables is dependent on. Most could be determined directly from the system's configuration (e.g. the number of sensors $\mathrm{N}$ ), through simple measurements (e.g. the directory service delay d), or by estimation (e.g. the average cross-sector distance $\sqrt{S}$ ). The degradation constant $\lambda$ required a more detailed performance evaluation to find an appropriate value. In practice, if a complete prototype is not available to make a determination, one could approximate such values through targeted simulation of the appropriate subsystem [8], through formal analysis of the algorithm or technique in question, or by using a backof-the-envelope estimate that is revised as additional data is available. Figure 10 shows a comparison of the previously observed number of measurements and the predicted obtained from Equation 7 using these values.

Although the detailed results are not presented here, similar analytic models were also created for estimating the load placed on track managers [7].

\section{UNIFIED ORGANIZATIONAL MODELING}

The analytic models presented in the previous section, although individually precise, lack the cohesion necessary to create a complete prediction of system performance. There is no strong notion that particular and distinct entities exist with associated characteristics. There is no well-defined way of specifying what decisions must be made, what values must be optimized over, or what constraints must be respected. Instead, such individual expressions provide performance characteristics piecemeal, and comparative analysis of entire systems is performed later in an ad hoc manner. For example, note the discontinuity between the measurement requests predicted by Equations 1 and 7 . While we could copy the appropriate logic into Equation 1, this duplication of effort is somehow dissatisfying, and the resulting equations would still fail to capture the underlying relationship at the root of the problem. Finally, while the provided equations are able to model the effects of a changing sector size, we believe a single, static set of equations will be unable to represent all the alternative ways that a structure might be created in a concrete and accurate manner. For example, consider if there were a choice of the type of sensor or agent available for use in the environment, or different tracking techniques that might be employed, or an optional information aggregation hierarchy of arbitrary height and width. While one could create individual models for each dimension, combining them together in a coherent and expressive way would be challenging. It is for this reason that we view tools that operate principally on such representations, such as nonlinear solvers and queuing networks, as too limiting for our purposes (although we believe they may play a role in certain aspects of design evaluation).

To address this deficiency, we have developed a more robust set of tools to capture organizational information in a single, unified structure. The Organizational Design Modeling 
Language (ODML) provides domain-independent mechanisms to model, evaluate and compare a variety of organizational styles, including the sensor network we have described in this paper. As we will show, ODML incorporates quantitative information in the form of mathematical expressions similar to those used above. These expressions are grouped into organizational constructs, connected via a graph of relationships, and ultimately used to represent and predict both the localized and global characteristics of an organization.

The immediate benefits of such a language are twofold. First, by incorporating quantitative information about the environment, resources, agents, tasks, goals, or any other object relevant to the system's performance, candidate organizations may be tailored and evaluated in a context-specific way. For example, we may directly embed information about sensor density, target velocity, communication limitations, and the like. This model can then be used to determine the organization which is most appropriate for that context, given a particular definition of utility. Second, once a suitable model hás been found, it can serve as an explicit organizational representation, guiding agents' local decisions in a manner consistent with global objectives. The longer-term benefits of the organizational model include being able to make predictions about runtime performance, which can be used to isolate and diagnose system failures and deficiencies. This same information can also be used to support adaptation of the system, by incorporating learned knowledge into the existing model and analyzing the resulting structure.

\subsection{ODML}

An organizational model, as we envision it, serves in several different capacities. At design time, it should be possible to use the structure to create and evaluate not just a single organizational instance, but an entire family of organizational possibilities. At runtime, it should accurately describe the current organization. In both cases, the model must be sufficiently descriptive and quantitative that one can evaluate the organization's effectiveness, and rank alternatives according to some specified criteria. Below, we enumerate the desired capabilities and characteristics a modeling language should possess to satisfy our requirements:

Represent a particular organizational structure. This would include roles, interactions and associations (e.g., coalitions or teams). Different flows in the organization, such as communication and resources, should be representable.

Represent the range of organizational possibilities, by identifying general classes of organizations and the parameters that influence their behavior. Different elements should be modelable at different levels of abstraction. Identify which characteristics are under deliberate control, and which are derived from external factors.

3. Enable concrete performance predictions and allow deductive analysis by quantitatively describing the relevant characteristics exhibited by the structure, the manner in which those characteristics interact, and the constraints they are affected by. For example, both communication overhead and the effect that overhead has on work load should be representable.

Many different organizational representation schemes have been developed by researchers $[1,2,3,4,9,12,16,17,18,19$, 24]. Most, if not all these representations can satisfy the first two points to varying degrees, but none are able to incorporate quantitative knowledge in such a way that concrete, organization-centric predictions can be made directly from the model itself. In this section we describe a new formalism called ODML that explores how such information can be modeled and used.

Most existing representations fall into one of two categories: either they represent a wide range of organizational characteristics abstractly, or they can capture a smaller set of characteristics concretely. The former are usually good at representing what entities or relationships exist or could exist, but cannot compare alternatives in a quantitative way. The latter may contain quantitative knowledge, but have difficulty relating that knowledge to specific organizational concepts, mitigating their usefulness if one is hoping to understand the effects a particular organizational design will have.

For example, OMNI [3] and [9] can each capture a greater variety of organizational concepts than ODML, but do so in a largely qualitative way. Conversely, both SADDE [18] and MIT's Process Handbook [16] can incorporate arbitrary quantitative information, but neither couples this information with the organizational structure in a way that enables one to deduce how the characteristics of one aspect of the design affect another. Decker's TAEMS representation [1] does directly embed a certain amount of quantitative information, but this data is abstract and can only be used to make detailed performance predictions of a limited set of characteristics. The representation created by Sims [19] is perhaps closest in purpose to ODML. It incorporates detailed quantitative information into a structured organizational model, but does not have the innate ability to evaluate and rank organizations based on this information. We also believe ODML's more flexible design can model more situations at different levels of abstraction.

The benefits offered by ODML do not come without their price. For example, the flexibility alluded to above can result in a vast number of alternative designs. This, coupled with a general paucity of strong organizational landmarks on which heuristics could be founded conspire to make the search for designs a potentially difficult task. In the end, each representation has its strengths, and ODML's goal is not to supplant these works - but to demonstrate another approach that makes different tradeoffs. As shown below, ODML does so by incorporating a concrete but flexible set of primitives that can model a range of organizational constructs along with the quantitative characteristics that differentiate them.

We continue by formally defining an ODML template specification as follows:

$$
\begin{aligned}
\mathcal{O} & =\{\mathcal{N}, H, C, K, M, V\} \\
\mathcal{N} & =\left\{N_{0}, N_{1}, \ldots, N_{n}\right\} \\
N_{i} & =\{t, \ell, \bar{p}, I, H, C, K, M, V\}
\end{aligned}
$$

The foundation of the ODML template specification is the set of node templates, each of which corresponds to a particular physical or logical entity which might exist in the organization. For example, in our sensor network scenario there would be nodes corresponding to sectors, managers, relationships, agents and the environment, among other things. Each node $\mathrm{N}$ contains a number of elements, defined below:

$\mathrm{t}$ The node's type. This label must be unique within the set of template nodes that make up the organization.

$N \cdot{ }^{-}=\langle$symbol $\rangle$

$\forall N, M \in \mathcal{N}, N . t=M . t \Leftrightarrow N=M$

a. The node's instance limit. This specifies the maximum number of instances of the node type permitted in a valid organizational instance.

\section{$N . \ell \in\left\{\mathbb{Z}^{+} \cup \infty\right\}$}

b. An ordered list of parameters that must be passed to the node's template when an instance of the node is created. These are analogous to the parameters one might pass to an object constructor. Each parameter is specified with a type and local name.

$N \cdot \bar{p}=[\langle$ symbol, type $\rangle, \ldots]$

I The set of node types that this node has an is-a relation with using conventional object-oriented inheritance semantics. If we assume that a node's $\mathrm{I}=\{\mathrm{a}$, b $\}$, an instance of the node will also be an instance of $\mathrm{a}$ and $\mathrm{b}$, possessing the characteristics of all three node types. Isa relationships cannot be cyclic, i.e., $\mathrm{N}$ cannot have itself as a decedent.

$N . I=\{\langle t y p e\rangle, \ldots\}$

$\forall i \in N . I, N \neq i \wedge N \notin i . I \wedge \ldots$ 
$H$ The set of node types that this node has a has-a relation with. If we assume that $H=\{a, b\}$, an instance of the node will possess some number of instances of both $a$ and $b$. It is through this type of relationship that the primary organizational decomposition is formed. Each hasa has a magnitude that specifies the number of instances connected by the relationship.

$$
\begin{aligned}
& N . I I=\{\langle\text { symbol, type, magnitude }\rangle, \ldots . \\
& \text { magnitude }=\langle\text { symbol }\rangle
\end{aligned}
$$

C A set of constants that represent quantified characteristics associated with the node. Constants may be defined with numeric constants (e.g., 42), or mathematical expressions (e.g., $x+y)$.

$$
\text { N.C }=\{\langle\text { symbol, expression }\rangle, \ldots\}
$$

K A set of constraints. Also defined with expressions, na organization can be considered valid only if all of its constraints are satisfied.

$$
\begin{aligned}
& N . K=\{\langle\text { symbol,op, expression }\rangle, \ldots\} \\
& o p \in\{\langle,\rangle, \leq, \geq,=, \neq\}
\end{aligned}
$$

M A set of modifiers that can affect (e.g., mathematically change) a value contained by a node. Multiple modifiers may affect the same value. Modifiers model flows and interactions by allowing the characteristics and decisions made in one node to affect those of another.

$$
\begin{aligned}
& \text { N.M }=\{\langle\text { symbol,op, expression }\rangle, \ldots\} \\
& o p \in\{+,-, \times, \div\}
\end{aligned}
$$

$\checkmark$ A set of variables, representing decisions that must be made when the node is instantiated. Each variable is associated with a range of values it can take on. For example, a node might have a variable $x$ that could take any one value in the set

$N . V=\{\langle$ symbol, $\{$ expression,$\ldots\}\rangle, \ldots\}$

symbol refers to a user-defined string, similar to a variable name in a conventional programming language. These typically describe or refer to a particular characteristic. Type is the type name of some defined node, so such that N.t = type. expression is an arbitrary algebraic expression, possibly referencing constants, symbols and function calls. ODML supports the use of floating point values, lists of floating point values, and discrete probabilistic distributions in these expressions.

The top-level organization node also contains the elements $\mathrm{H}, \mathrm{C}, \mathrm{K}, \mathrm{M}, \mathrm{V}$, providing a location to embed additional global information and constraints. Collectively, we refer to $\mathrm{C}$, $\mathrm{K}$ $\mathrm{M}, \mathrm{V}$ as a node's fields, and the quantitative state of a field as its value. For example, a constant field total load might be defined with the expression total_load $=$ work_load + communication_load and have a value of 0.9 for a particular agent. Note that the use of the term "constant" may initially be misleading. While the expression defining total_load is fixed, the value for total_load produced by that expression may change through the application of modifiers, or due to changes in fields or values that the expression is dependent on.

At first glance, the ODML language may appear to be devoid of almost all the organizational concepts that are provided by typical organizational representations. This is partially true, and by design. Instead of directly incorporating the usual high-level organizational components, such as hierarchies, roles, agents, etc., ODML provides a set of relatively low-level primitives by which such structures can be defined. For example, a node with the user-defined type manager, having a has-a relationship with another node of type agent could embody a role-agent relationship. A sequence of has-a relationships between nodes could indicate a hierarchy.

Although the high-level semantics for these nodes may only be implicit, the concrete characteristics and design ramifications are still directly and quantitatively captured by the nodes' fields. We feel that this approach can lead to an increased diversity of representable structures, by avoiding the assumptions and inevitable restrictions that typically accompany high-level structures.

ODML instances are quite similar to ODML templates. The difference is that where a template is a description of what could be, an instance is a description of what is. Where a template might specify that a manager role can be assigned to a single agent or distributed across multiple agent nodes, na instance would indicate that manager 1 is distributed across agent_5 and agent_7, and so on.

Instances are created by making choices for the decision points embodied in the template. Such decision points are captured in two different ways: in the choice of value to assigned to a variable field, and in the choice of node type to satisfy has-a relationships. Although employing just these two choice types may seem limiting, we have been able to use these simple concepts to capture many types of organizational possibilities. For example, a variable could be used to express the range of possible sensors per_sector in the DSN domain, to controls the shape of part of the organization. Other uses of variables might be to decide the relative priority of an agent's tasks, the amount of time it is willing to wait for a response, or the number of agents that will be used to form a coalition. Decisions made for the agent hasa relationships in the three roles will determine the specific role-agent bindings that will be used. Sequences of similar decisions could also decide if the manager role will be distributed, or how tall a data processing hierarchy should be. Deciding upon the correct decisions can be viewed as a search process, which is a subject of ongoing work. Once an instance has been created from these decisions, the expressions defined by the fields, the data passed in through parameters, and the interactions caused by relationships can all be used to predict values for an individual node's characteristics.

The formal definition of an instance is nearly identical to that given in Equation 8, so we will not repeat it here. The differences principally relate to the replacement of node types in the template with instances of those nodes in the organizational instance. Thus, the set is the set of node instances, whose individual types no longer need be unique. So, where there might be just a single manager type in the template, there can be an arbitrary number of manager instances in the instance. Both is-a (N.I) and has-a (N.H) relationships no longer reference node types, but particular node instances in . Finally, the set is filled with appropriate values from each node's parent, and the variable set $\mathrm{V}$ for each node is replaced by a single item from that variable's range. Because a common syntax is shared between the two forms, for the remainder of this document I will indicate where necessary which is being considered.

As mentioned above, it is the ability to use an ODML model to deduce quantitative values for specific characteristics that sets it apart from other representations. The manner in which these values are determined for an instance node's characteristics is defined by the pseudocode in Figure 11. Note that some aspects of get value's behavior, such as the manipulation of list-based data, have been omitted for clarity. This function shows how various sources of information, non-local data and node interrelationships all interact to describe the features of a particular node. It is through the execution of this function on a particular symbol that predictions are made of the design's performance. For example, agent.get_value(total_load) would return a prediction of agent's total_load. 


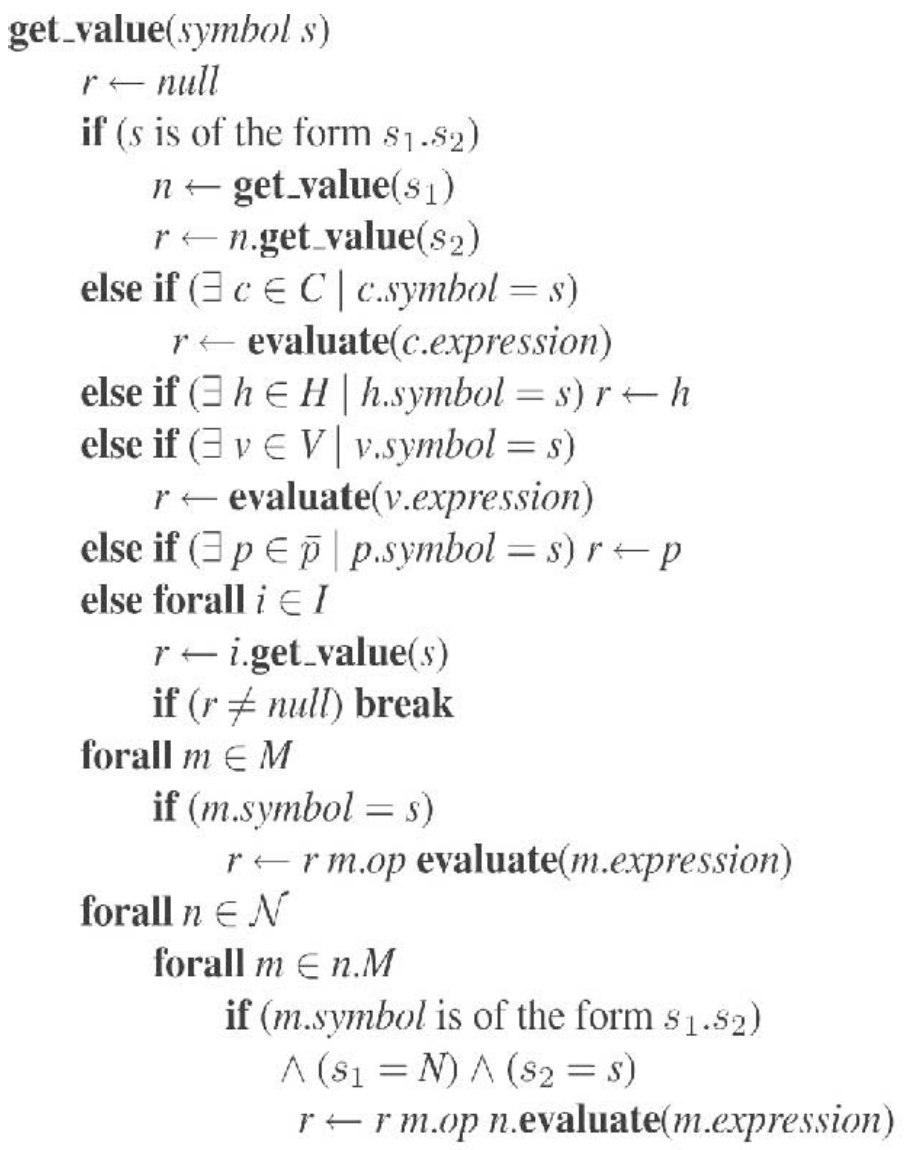

\section{return $r$}

\section{evaluate(expression e)}

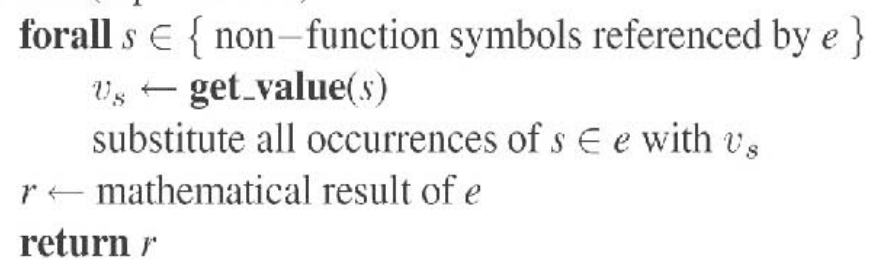

Figure 11 - Pseudocode for the get_value function of a node $N$. This function is used to quantify the caracteristics of instance nodes

\subsection{SENSOR NETWORK MODEL}

The capabilities of ODML are best explained through na example. We will proceed with an overviewof how an ODML model was produced for the distributed sensor network domain described in Section 2. For clarity, we will represent particular nodes, or fields that reside in the nodes, in italics. Space precludes showing the complete textual model constructed for the sensor network, however, a portion of the model can be seen in Figure 12. The complete model is roughly 280 lines long, including whitespace and comments. A corresponding graph showing some aspects of the model's template can be seen in Figure 13a. Vertices in that graph, such as sector and sensor, represent nodes. Nodes can represent both tangible (e.g. agent) and intangible (e.g. sector) entities. Directed edges with a solid arrow represent has-a relations, and the corresponding label indicates the magnitude of that relation. For example, each track manager node has a number of agents defined by the field num agents. The corresponding definition is shown in line 4 of Figure 12.

A hollow-arrow edge represents an is-a relation, so normal_agent is an instance of agent. Shaded nodes, such as agent are abstract, and cannot be directly instantiated. Thus, any node with a has-a relation with agent can instead substitute normal_agent. This level of indirection allows this model to represent and easily use agents with different capabilities. For example, the robust_agent mentioned in Section 4 is represented with a node that also has an is-a relation with agent, and can be substituted for agent in the same way. 
of a non-local modifier from the source node to a field in the target node. Black directed edges represent hasa relationships, but unlike the template they have no labels. Because this is a particular instance of the sensor network organization, the decision points present in the template have all been decided. Therefore, where sector might have the num_sensors label on its sensor relationship in the template, a discrete value of two has been chosen for that field in this particular instance. Because of this, each sector in the instance has two sensors (S). Normal agents (a), sector managers (SM), track managers (TM), and two kinds of track manager relations (SM-TM and S-TM), are also present.

We can relate this model directly to the organizational structures discussed in Sections 3.1 and 3.2. Geographic coalitions are embodied in the sector node. The size of the has-a relation sector has with the sensor node reflects the chosen sector size, and the sector manager is specified with the sector_manager node. The functional differentiation aspect is modeled directly by the sector_manager, track_manager and sensor nodes. Each represents a role that can be assigned. This assignment is represented with the agent has-a relationship each node possesses. The particular instance of agent node associated with a role node corresponds to the particular agent assigned to that role.

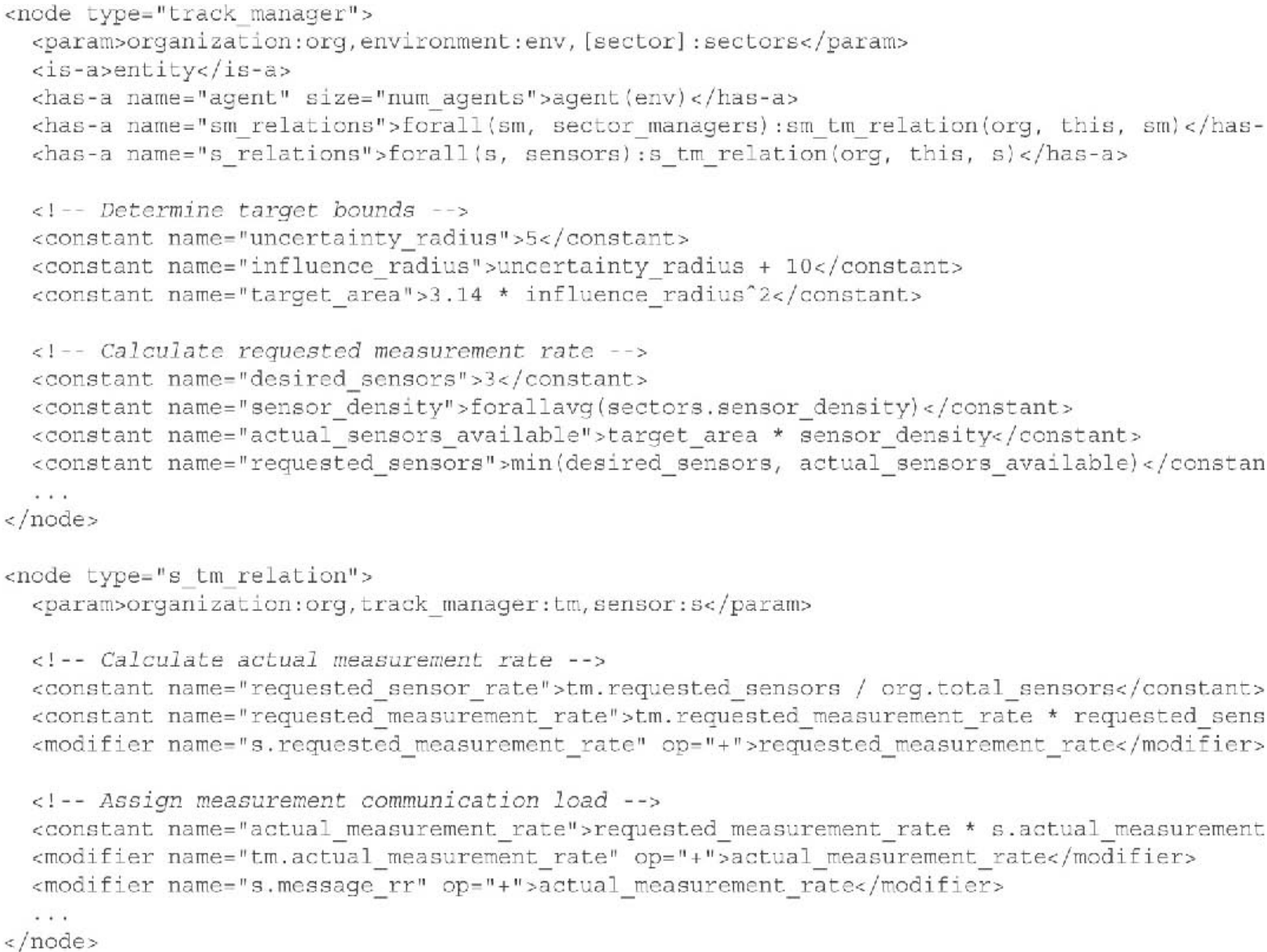

Figure 12 - A portion of the raw ODML specification for the track_manager and $s_{-}$tm_relation $\mathrm{n}$ 


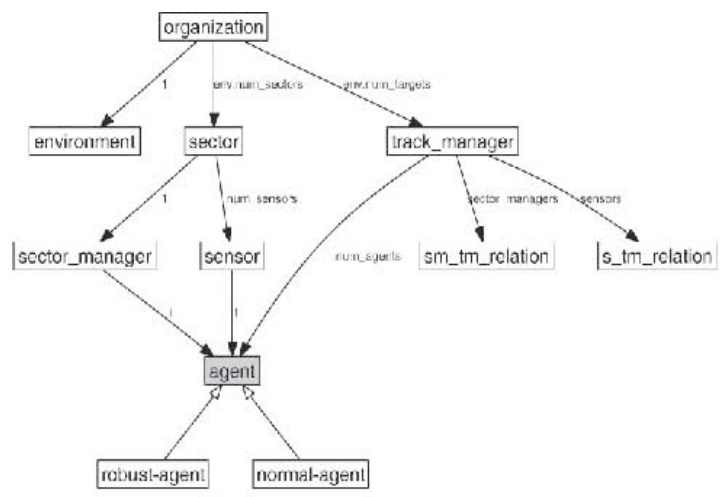

(a)

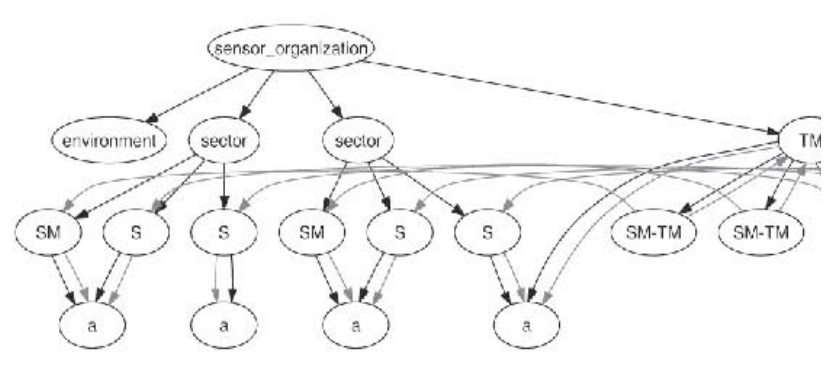

(b)

Figure 13 - Example ODML (a) template and (b) instance structures for the sensor network or:

The heart of any ODML model exists in the expressions encoded within nodes' fields. A selection of these fields, contained by the track_manager and $s$ tm_relation nodes, are shown in Figure 12. The former defines the track manager role, while the latter represents the relationship that role hás with sensors in the environment. Each node's field may contain an arbitrary mathematical equation, combining local and nonlocal information to calculate new local values as depicted in Figure 11. These expressions provide a way for the designer to represent how different characteristics of the node may be computed. For example, suppose we wish to define how to calculate the track manager's logical footprint (area) of a target as it moves through the environment. This área will depend on the amount of uncertainty the manager hás in the target's location, along with a factor modeling the target's "area of influence", that relates to the effective sector size discussed in Section 4. In our model, this area will be a circle; line 11 shows how the target_area of a track manager is derived from the target's influence_radius. The number of sensors presumed capable of sensing the target is the average number of sensor which lie within the target area. Therefore, although the number of desired_sensors is independent of the environment, the actual_sensors_available to the manager will depend indirectly on the target_area and sensor_density, as shown in line 16. The requested_sensors will be the minimum of the desired and available.

We may model the number of measurements provided to the track manager in a similar way. The actual measurement rate in the sensor-track manager relationship is derived from the locally calculated requested measurement rate and actual measurement ratio computed by the sensor node. This value is then used in a pair of modifiers defined in lines 31 and 32 that specify for the track manager and sensor the actual number of measurements that will be taken.

In this way, the characteristics of one node may affect or be affected by those of another. Oscillations and infinite recursion are avoided by allowing only acyclic equation relations. The resulting web of equations allows one to model important concepts such as information flow, control flow, and the effects of interactions. By propagating data through these expressions, the model can predict the characteristics of both individual nodes and the organization as a whole. Perhaps more importantly, it also allows the model to predict characteristics not necessarily envisioned or considered by the designer, as the results of expressions can flow through the graph in unanticipated ways. It is this automatic propagation which differentiates an ODML model from a simple set of equations, by creating a unified view of the complete working organization.

The mechanisms provided by the ODML primitives allows one to model a range of common, organizationallyinfluenced system characteristics. To continue our example, we will describe several such characteristics relevant to the sensor network organization, and demonstrate how the interplay between such elements results in a more coherent, unified model.

5.2.1. Environmental and System Constants Incorporating numeric constants within an ODML structure, a crucial element of any realistic model, can be at once simple to accomplish and difficult to complete successfully. The definition itself, comprised of a straightforward constant field, is trivial to create. Determining what value to place within this field can be an entirely different matter, just as with the values used in the discrete analytic models. For example, the desired_sensors constant at Figure 12 line 14 is a known quantity that can be extracted directly from agent code or a software engineering specification. On the other hand, the uncertainty_radius on line 9, which represents the expected radius of the target's uncertainty bound, can be more difficult to determine directly. Assuming for the moment that this value does not depend on other characteristics (such as the target's velocity), one could first specify a rough estimate, and later revise that estimate if contradictory empirical evidence is observed in practice or a more accurate value is devised. In practice, most of the numeric constants in the sensor network model were derived through a combination of known system parameters, estimation based on domain expert knowledge, and in some cases, instrumentation of a running system or prototype.

The specification of expression-based constants can be accomplished in a similar fashion, although these are more frequently determined based on knowledge of the system in question. An example of this is the track manager's requested_sensors in line 17 of Figure 12. This represents the number of sensors that manager will actually ask for, which may be less than desired sensors in the case where there is insufficient sensor density in the environment. It is sometimes the case, however, that a simple closed-form solution is either difficult to derive or not possible. In the former case, we have used curve-fitting techniques to obtain approximate expressions from empirical data. This techniquewas used to find a predictive expression for RMS error, based on the number of received measurements. It is worth noting that this particular expression attempts to abstractly and indirectly capture a number of complex effects, including the effects of target ambiguity, incorrect data fusion and the average quality of the measurements themselves.

For the latter case, when a closed-form solution does not exist or cannot be found, ODML supports a general "mapping" function, which allows one to define a function correlating a discrete input value with an arbitrary expression. With this, one may define some f such that, for example, $f(1)=x+\sqrt{y}, f(2)=32 z^{3}$, etc. The average effective_area of the sector nodes uses such a function. This characteristic, originally defined as in Equation 2, represents the average area covered by the sensors in each sector, which is typically larger than the area of the sector itself. A mapping function was used to effectively create a look-up table, which associates an appropriate expression calculating the effective area for each sector size.

5.2.2. Agent Interactions The manner in which entities interact is perhaps the most visible and defining characteristic of multi-agent systems. It generally plays a crucial role in determining how information flows through the system, how load is distributed, how efficient operations are, and ultimately the effectiveness of individual agents and the system as a whole. In our sensor network model, these interactions are defined in two different ways. In the first, entities simply model the effectsof interactions internally. For example, the sensor node represents the directory service messages it sends to its sector manager, and uses a modifier to add a corresponding number of messages to its sector manager. We have used both modifiers and parameters to disseminate such agent characteristics so that they may be incorporated in remote nodes.

The second technique employs a more explicit representation, by creating an independent node to model the interaction itself. An example is s_tm_relation, the sensorto-track manager relation, shown in Figures 12 and 13. This node models the interactions that take place between a track manager and a sensor, which include determining the rate at which task requests are generated by the track manager, the rate at which measurements are taken in response to those requests, and the rate at which corresponding results are sent back to the track manager. Each of these values is calculated using a combination of information from each entity, and the results applied back to the appropriate node. For example, the actual_measurement_rate is used to increment the sensor's messages_rr constant, which tracks the number of result messages that are sent. 
creation. We have found that there are several ways that more complex interactions benefit from an explicit, separate model. By separating and encapsulating the interaction, its effects can be made more transparent and the model more understandable. In the case where there is a one-tomany type of relation, as with a track manager and the sensors it uses, this type of separation also facilitates the expression writing process by limiting the scope that individual equations must cover. Finally, in the case where several alternative interaction styles are available, the explicit representation allows the designer to use variables or inheritance to model and reason about such choices. For example, if our track managers had two different ways of requesting measurements from a sensor, those alternative interactions could be modeled as s_tm_relation1 and s_tm_relation2, each defined as an instance of s_tm_relation with an is-a relationship. When an instance of track_manager is created, one of those two alternatives would be selected for each sensor, and the corresponding effects incorporated appropriately. In this way, in addition to representing the quantitative effects of interactions, the selection of agent interaction or coordination mechanisms may be cast as an organizational decision in ODML.

5.2.3. Multiple Role Assignments In human organizations, individuals frequently act in many different capacities, serving different needs and exhibiting different behaviors depending on the working context. In some complex multi-agent systems, similar phenomena may be observed, where individual agents take on multiple roles that dictate the various responsibilities, capabilities and activities it is associated with. Because the assignment of these roles to individual agents is na organizational decision, it is important represent both the assignment itself and the cumulative effects of that decision.

As mentioned earlier, there are three roles in our distributed sensor network organization: the sector manager, the track manager and the sensor. These are represented by the sector_manager, track_manager, and sensor nodes, respectively. Role assignment is modeled through the use of a hasa relationship. Specifically, each of these role nodes has na agent, as shown in Figure 13a, that represents the particular agent that role is assigned to. During instantiation, has-a relations may be fulfilled in two different ways. Either a new instance of the target node is created to satisfy the relationship, or an existing instance of the node is used in the same way. Na example of the latter can be seen in Figure 13b, where the leftmost node $a$ is owned by both SM and S, indicating that particular agent has been assigned to two roles.

Most of the detailed characteristics in this model are computed within the role nodes. Therefore, important aspects such as load and resource usage are inherently separate and rolespecific. To capture the effects of multiple role assignments, these individual characteristics are first propagated into their relevant agent using modifiers. Each agent can then predict the cumulative effects of its roles. A natural example of this in this is the propagation of communication effects, which we have mentioned earlier. In this case the communication load of the agent is computed to be the sum of the communication loads of the roles it takes on. If we wish to capture more complex situations, such as superor sub-additive effects, the the agent load can be defined as some function of the various role loads that correctly accounts for those effects. This combined load can then be accessed and utilized by the individual roles in whatever manner in appropriate.

This confluence also provides a useful place to incorporate constraints that might be affected by multiple roles. For example, a design assumption in the original system said that each agent would be associated with a single sensor. In the ODML model, each sensor role uses a modifier to increment the sensors_controlled field of its agent. We may embody the design assumption by placing a constraint in the agent, specifying that sensors controlled must be equal to 1 , which guarantees that all agents in a valid organization will control exactly one sensor. This also demonstrates one way to control how many roles an agent is assigned. A similar approach could constrain communication or processing load which tie role assignment to a more concrete metric. Conversely, by adding a constraint defining a lower bound on load, we can make the selection process more conservative by ensuring all created agents see a certain minimum level of work.

5.2.4. Dynamic Role Assignment In reactive or adaptive systems, roles are frequently created dynamically in response to emerging phenomena. Such is the case with the track manager role, which is assigned only when a new target has been detected in the environment. Although we can and do model rates of change and expected value, there is no explicit representation of a varying timeline or change points in the model. Therefore, ODML instances generally represent a snapshot of a running system, or an averaging of effects as they would occur over some span of time. If dynamic elements exist in the source environment or system, they may be represented in that same manner. For example, although at any given point in time there may be many or few targets in the environment, there is some expected number of targets that represents a statistically average value. This number would then be used to estimate the "normal" situation, and be reflected in the model accordingly. ODML also natively supports the use of discrete probabilistic distributions, allowing one to explicitly represent a finite set of possible conditions. Furthermore, because ODML is based on sets of arbitrary equations, one could also use continuous distributions (e.g., Poisson), provided the means to analyze them can be expressed using closed-form expressions. For example, by encoding the appropriate parameters as constants (e.g., $x, \lambda)$ and then manipulating those values using traditional queuing theory techniques [10], one can integrate and estimate behaviors based on these more complex assumptions.

Additional dynamism is present in the sensor network example, due to the migration of the track manager role as described in Section 2 . When this role moves to maintain locality with its target, the effects of that role are effectively spread over multiple different agents. To represent this effect, the model uses the target's velocity and the sectors' sizes to first estimate how frequently that role will migrate. Because this is a rate, it must be combined with the duration of the scenario to determine the number of agents that role will be assigned to. This number is then used to calculate num_agents, which as mentioned earlier is used to specify the size of the track manager's agent has-a relationship. So, if the model predicts that the track manager role will be created and then migrate twice, the num agents field in track manager will be set to three. The role's relevant characteristics are divided and distributed evenly among those three agents using modifiers as described previously.

5.2.5. Heterogeneity Another important advantage that ODML offers over the simple analytic models from Section 4 is that heterogeneity is more readily representable. When calculating the total number of measurements for a track in Equation 7, for example, we assumed that all sensors would produce measurements at equal rates. Similarly, the sector manager's load in Equation 2 assumed that all targets moved with equal velocity. Neither of these simplifying assumptions are likely to be true in practice, so to the degree the unified model can represent such additional information, it will have a decisive advantage in accuracy.

ODML's ability to model heterogeneity is derived from the the node-based representation of entities. Because each role, agent or other structure is defined as a separate node in the organizational instance, entities that share a common type may still contain different values or be affected by different organizational pressures and flows. We have seen examples of this in the previous two sections. Because agents may be assigned single or multiple roles, the resulting agent population has the potential to be heterogeneous in the final organization. These variations may then propagate through the organizational instance to create differences elsewhere in the model. For example, communication load can be tied to the agent's capacity to perform work, which could affect the number of measurements its sensor role could take, which would affect the RMS error of the track manager using that sensor.

Inheritance provides an additional mechanism to represent heterogeneity. For example, to model the "robust managers" scenario from Section 4, we defined a robust agent node that has an is-a relationship with agent. This effectively creates two different classes of agents that can be employed, each with potentially different capabilities and costs.

5.2.6. Conflicts, Constraints and Resolution Many of the more interesting aspects of organizational models revolve around the limits or constraints that are imposed on the system and what happens when those limits are approached or exceeded. ODML models can represent both hard and soft constraints. The former include conditions which the designer has deemed untenable, while the latter are usually characteristics that degrade more gradually, and may be tolerated by the system.

Hard constraints may be modeled using constraint fields, as described in Section 5.1. A constraint is defined with a target, a relational operator, and an expression. To verify the constraint, both the target and the expression are evaluated to produce numeric values, which are then compared with the provided operator. The constraint is considered satisfied if the resulting relation is true, and unsatisfied if otherwise. Because a valid organization must contain only satisfied constraints, they are considered hard, or strict conditions that must be met.We mentioned earlier how a constraint on the sensors_controlled field in the agent ensured a one-toone mapping between sensors and agents. Similar constraints could be added to set an upper bound on average expected RMS, a limit on local work load, or a maximum number of agents in the organization. Although our sensors were hardwired, a battery-driven sensor network could also be modeled by adding a suitable constraint to the agent. In this case, communication rates, action rates or the passage of time could decrement a battery constant. A constraint placing a lower bound on the battery would ensure that the unit met an expected minimum performance.

Soft constraints have a more subtle effect on the system. They are not explicitly modeled using the constraint field. Instead, we represent them using equations that affect performance in response to other attributes. For example, in the sector manager, excessive communication load can delay directory service responses. This is modeled with a directory_delay field, which is then used to determine the values for fields in the track manager analogous to the $d$ and $g$ delay values of Equation 7 . Increases in those values will eventually increase the RMS error by slowing the rate at which the track manager acquires new sensor information. This is represented in the model by using a modifier to reduce the track manager's requested_measu-rements_rate in response to increased directory delays. Therefore, although there is no fixed, arbitrary limit on sector manager load, excessive load will still degrade the system's performance.A breaking point, at which the performance level has become untenable, can still be modeled using a hard constraint governing the value in question. 
Soft constraints are also used to model the competition for sensors by track managers. The s_tm_relation in Figure 12 notifies each sensor of the requested measurement rate that will be asked of it. If the requested rate exceeds the sensor's capa bilities, the actual_measurement_rate will be lower, reduced to some fraction of the possible rate proportional to what was asked for. This will negatively affect the expected RMS error. So, although there is no set limit on the number of track managers, a "tragedy of the commons"- style degradation in quality can be predicted from overusage [25].

\subsection{MODELING RESULTS}

To gauge the efficacy of the ODML representation, we have constructed the model described in the previous section, used it to create organizational instances that match the prior test runs, and compared the predicted characteristics against the empirical results from Section 3. Because timebased characteristics in the ODML model (such as communication load) are computed as rates rather than totals, the values are not directly comparable. However, cumulative totals may easily computed by multiplying the relevant rate by the length of the prior simulation run.

A relatively coarse set of comparative results are shown in Figure 14, which contrast the predicted results against some of the actual, empirical results shown earlier in the paper. Solid lines represent the values predicted by the ODML model, while dashed are those obtained through empirical testing. Although there are a few significant points of difference, in most cases, the model does a good job predicting performance. One difference can be seen in Figure 14b, where the predicted standard deviation underestimates the actual performance in most cases. This is a byproduct of our assumption that all sensors were equally used. In the running system, sensors in the center of the environment are used more than those at the edges, and will have different communication profiles because of it. Our model does not capture these geographic differences, and will therefore generally have a lower estimated deviation. The analytic models in Section 4 suffer from this drawback as well.

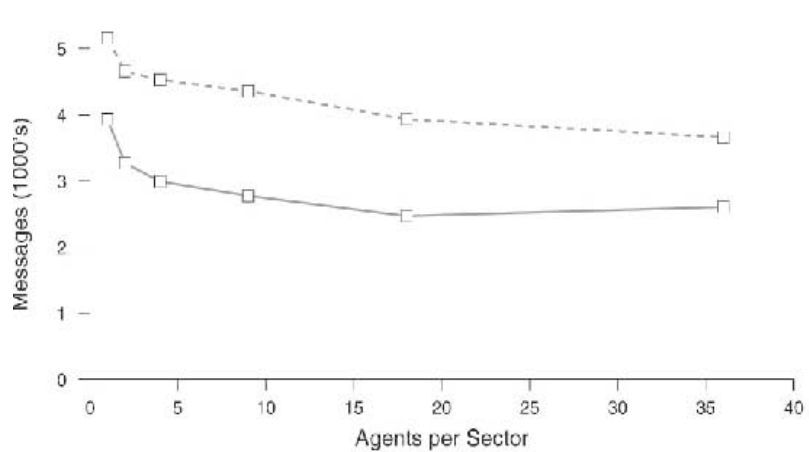

(a)

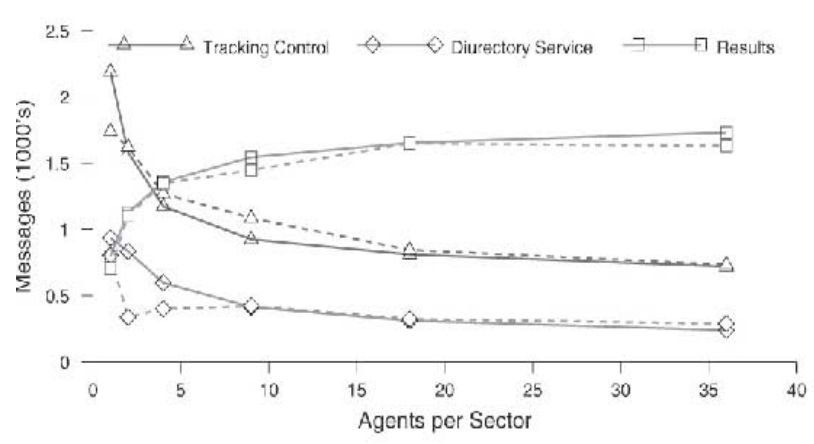

(c)

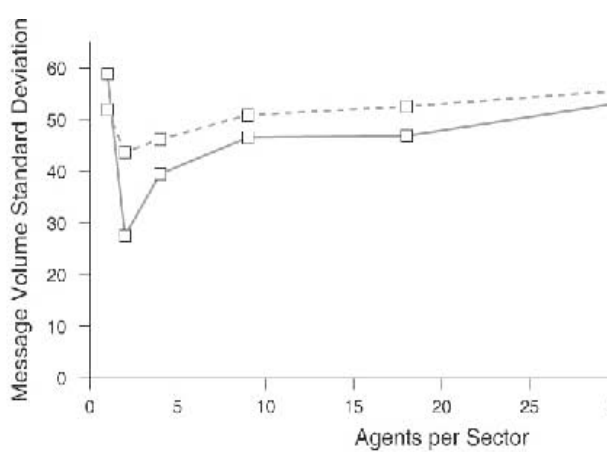

(b)

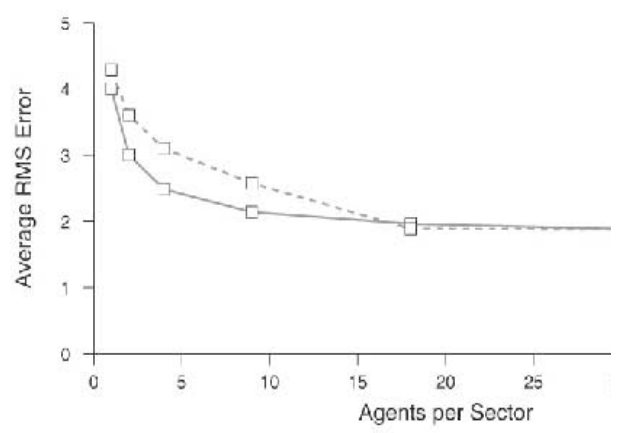

(d)

Figure 14. Performance predicted by the ODML sensor network model versus empirical observa for a) Total messaging, b) Messaging disparity, c) Message type totals and d) RMS error. Predi lines are solid, empirical are dashed.

A more obvious difference can been seen between the overall message totals, in Figure $14 a$. This difference can be attributed to the fact that the empirical values included all 24 message types that occurred in the system, while the model only tracks the five most significant message types. Combined, those five types constituted roughly $80 \%$ of the communication volume on average. The remaining 19 were uncommon; no individual type accounted for more more than $3 \%$ of the total. As can be seen in Figure 14 a, the difference between predicted and empirical remains relatively constant with sector size, and could be accounted for by adding a suitable constant to the model. The exclusion of these message types was a conscious choice on our part. It is an example of trading off the complexity of the model with its fidelity.

Recall that one of our initial goals was to predict organization-level characteristics of the system. The met rics we have shown so far accomplish this, but primarily on a global, aggregate level. To evaluate how our model predicts finer-grained details, we produced a separate set of graphs that show communication profiles by role, rather than the system-wide totals seen in Figure 14c. The actual and predicted role-specific graphs can be seen side-byside in Figure 15 . In addition to the communication totals we have discussed, these graphs also include role counts, indicating how many agents did or would take on that role in the environment. ' $A$ ' represents the sensor role, ' $M$ ' is the sector_manager, while ' $T$ ' is the track_manager. The role 'AT' describes agents acting as both sensors and track managers. These results are also encouraging. By and large, our predictions are similar to observations. Some of the differences, such as the result totals for some sector and track managers, can be attributed to geographic variances in a small sample size. For example, the 36- and 18-size sector scenarios had only one and two sector managers, respectively. Their individual geographic locations would certainly affect the 
averages in Figure 15c, and these variations are not reflected in the corresponding values in Figure 15d.

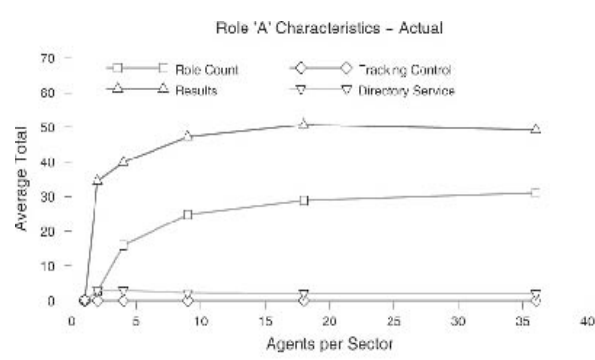

(a)

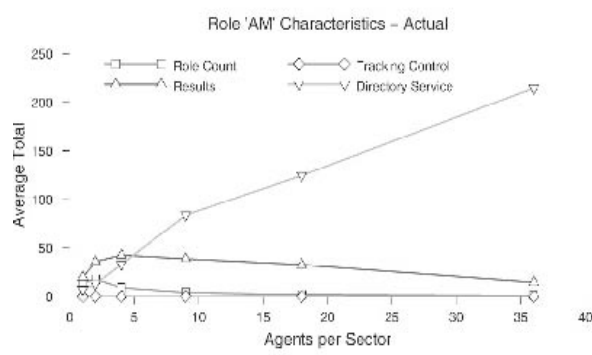

(c)

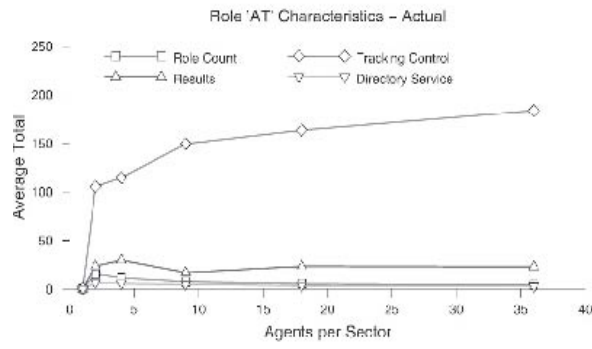

(e)

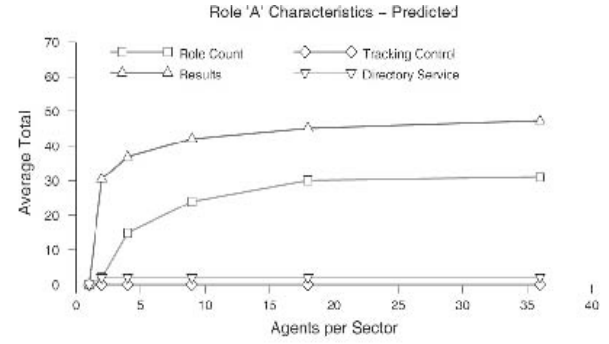

(b)

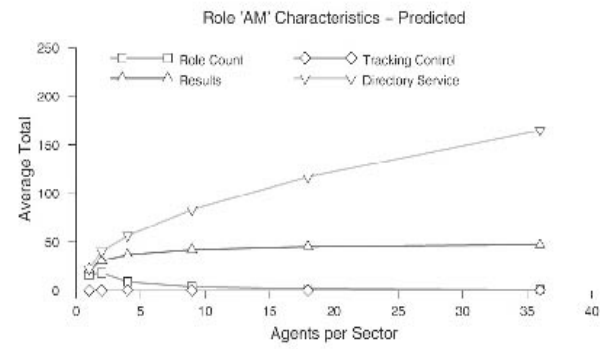

(d)

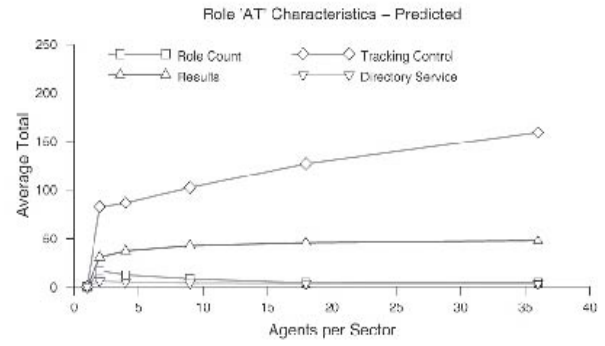

(f)

Figure 15 - A comparison of the average actual and model-predicted characteristics by role, for agents operating in the distributed sensor network

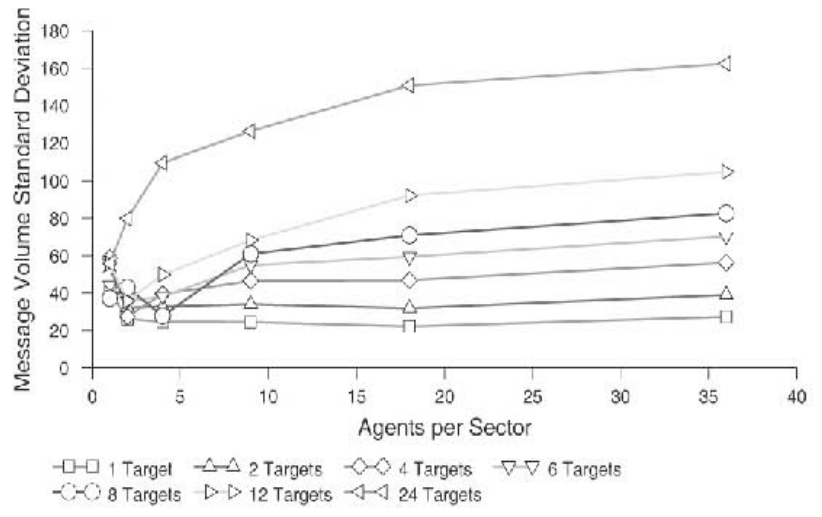

(a)

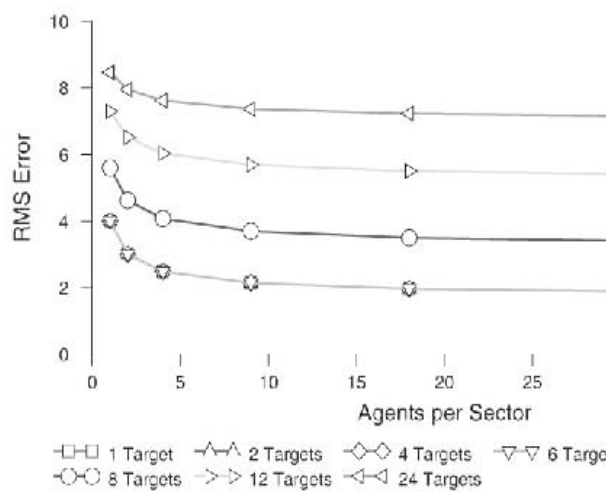

(b)

Figure 16 - Performance predicted by the ODML model as the number of target is varied for a) Messaging disparity, and b) RMS error 
Our last set of ODML predictions are shown in Figure 16, which examines messaging disparity and RMS error as both the sector size and the number of targets are varied. Figure $16 \mathrm{a}$ corresponds to the empirical results shown in Figure 7, while 16b corresponds to Figure 8 . The trends shown in those earlier Figures are similar to those predicted by ODML, including the same general profiles and the RMS phase transition. A notable exception is the 24 target case in Figure 7 a, which has a different profile than those previously observed. We believe this is the case because the volume of measurement messages generated by such a large target population dominated the contribution of the sector manager role when the sector sizes were relatively small.

\section{CONCLUSIONS}

In this article we have pursued two separate but related purposes. The first, discussed in Sections 2 and 3 demonstrated the role that organizations can play in when designing both sensor networks and multi-agent or distributed systems in general. We have also shown how the organizational design parameters can affect the system's performance in a variety of ways, both good and bad. It is our belief that any multi-agent system of even moderate complexity will have some form of organization embedded within it, even if it is only implicit.

This leads to our second purpose, covered in Sections 4 and 5.1, which is to motivate the creation and use of explicit models of organizations. We demonstrated that it is possible to create quantitative organizational models in ODML that accurately predict large and small scale performance. Such models can be used at design time to find and evaluate candidate organizations or identify design weaknesses.

An aspect of our future work is to demonstrate that ODML models may also be used at runtime to verify base assumptions and adapt the organization when necessary. For example, as new conditions become apparent at runtime (e.g. resource availability changes, environmental constants vary, etc.), these updated values can be inserted into the organizational instance. The instance can then be used to determine if constraints have been violated, and if so, the corresponding template can be searched for an appropriate reconfiguration. This is conceptually similar to the limited reconfiguration behavior of Tambe's STEAM framework based on structural properties [23], in which failure of an agent leads to a search for agents to take over the roles no longer served by the failed agent. Ultimately, we feel that the creation of more formal models of agent systems will foster an increased understanding of such systems, allowing them to be more contextsensitive and robust in the face of change.

The quantitative results we have presented are quite domain specific. They depend on the communication characteristics of the environment, the actions needed to achieve the scenario goals, and the behaviors exhibited by the agents. However, we feel that the types of issues raised by these particular experiments, such as information locality, specialization bottlenecks and organizational control overhead, are applicable to many different domains, particularly those which are communication intensive. For instance, our sector size results can be directly related to the estimated load incurred by a distributed collection of middle agents [22]. We have also shown in other work how ODML models can be applied to information retrieval in a peer-to-peer network domain [6], as well as more abstract, theoretical problems such as SUBSETSUM and TILINGS.

More generally, we feel that exploiting multi-agent organizations can have significant positive effects on performance, while avoiding some of the common pitfalls associated with scale. By specifying roles, authority relationships and working groups, the system can both reduce runtime combinatorics by restricting search as well as improve global coherence without requiring a global view. However, we have seen that these benefits come with costs and side effects, which must be understood for the organization to be used successfully. Formal models such as those provided by ODML can help make these tradeoffs concrete, and the quantitative comparisons they facilitate can guide the development process toward an appropriate selection of design and parameters. In this paper, we varied just one organizational parameter, and observed the ramifications of this change across several distinct dimensions. With continued research in this area, the complete space of organizational types and their corresponding characteristics can be more fully understood and exploited.

\section{REFERENCES}

1. K. Decker and V. R. Lesser. Quantitative Modeling of Complex Environments. International Journal of Intelligent Systems in Accounting, Finance and Management. Special Issue on Mathematical and Computational Models and Characteristics of Agent Behaviour., 2:215-234, January 1993.

2. S. DeLoach. Modeling organizational rules in the multi-agent systems engineering methodology. In Proceedings of the 15th Conference of the Canadian Society for Computational Studies of Intelligence on Advances in Artificial Intelligence, pages 1-15. Springer-Verlag, 2002.

3. V. Dignum, J. Vazquez-Salceda, and F. Dignum. Omni: Introducing social structure, norms and ontologies into agent organizations. In Second International Workshop on Programming Multi-Agent Systems at the Third International Joint Conference on Autonomous Agents and Multi-Agent Systems, pages 91-102, New York, NY, July 202004.

4. M. Fox, M. Barbuceanu, M. Gruninger, and J. Lin. An Organizational Ontology for Enterprise Modeling. In M. J. Prietula, K. M. Carley, and L. Gasser, editors, Simulating Organizations: Computational Models of Institutions and Groups, pages 131-152. AAAI Press / MIT Press, 1998.

5. M. S. Fox. An organizational view of distributed systems. IEEE Transactions on Systems, Man, and Cybernetics, 11(1):70-80, Jan. 1981.

6. B. Horling and V. Lesser. Quantitative Organizational Models for Large-Scale Agent Systems. In Proceedings of the International Workshop on Massively Multi-Agent Systems, pages 297-312, Kyoto, Japan, December 2004

7. B.Horling, R. Mailler, and V. Lesser. A Case Study of Organizational Effects in a Distributed Sensor Network. In Proceedings of the AAAI-04 Workshop on Agent Organizations: Theory and Practice, pages 23-30, San Jose, California, July 2004. AAAI Press, California.

8. B. Horling, R. Mailler, and V. Lesser. Farm: A Scalable Environment for Multi-Agent Development and Evaluation. In A. G. C. Lucena, J. C. A. Romanovsky, and P. Alencar, editors, Advances in Software Engineering for Multi-Agent Systems, pages 220-237. Springer-Verlag, Berlin, February 2004.

9. J. F. H"ubner, J. S. Sichman, and O. Boissier. A model for the structural, functional, and deontic specification of organizations in multiagent systems. In Proceedings of the Brazilian Symposium on Artificial Intelligence (SBIA'02), pages 118-128, 2002.

10. L. Kleinrock. Queueing Systems. Volume I: Theory. John Wiley \& Sons, New York, 1975.

11. J. H. Lawton. Distributed Sensor Networks: A Multiagent Perspective, chapter The Radsim Simulator, pages 11-20. Kluwer Academic Publishers, 2003.

12. V.Lesser, K. Decker, T.Wagner, N. Carver, A. Garvey, B. Horling, D. Neiman, R. Podorozhny, M. NagendraPrasad, A. Raja, R. Vincent, P. Xuan, and X. Zhang. Evolution of the GPGP/- TAEMS Domain-Independent Coordination Framework. Autonomous Agents and Multi-Agent Systems, 9(1):87-143, July 2004.

13. V. Lesser and L. Erman. Distributed Interpretation: A Model and an Experiment. IEEE Transactions on Computers Special Issue on Distributed Processing, C-29(12):1144-1163, December 1980

14. V. Lesser, C. Ortiz, and M. Tambe, editors. Distributed Sensor Networks: A Multiagent Perspective (Edited book), volume 9. Kluwer Academic Publishers, May 2003.

15. R. Mailler, V. Lesser, and B. Horling. Cooperative Negotiation for Soft Real-Time Distributed Resource Allocation. In Proceedings of Second International Joint Conference on 
Autonomous Agents and MultiAgent Systems (AAMAS 2003), pages 576-583, Melbourne, July 2003. ACM Press.

16. T.W. Malone, K. Crowston, J. Lee, B. Pentland, C. Dellarocas, G. Wyner, J. Quimby, C. S. Osborn, A. Bernstein, G. Herman, M. Klein, and E. O'Donnell. Tools for inventing organizations: Toward a handbook of organizational processes. Management Science, 45(3):425-443, 1999.

17. H. E. Pattison, D. D. Corkill, and V. R. Lesser. Instantiating Descriptions of Organizational Structures. Distributed Artificial Intelligence, Research Notes in Artificial Intelligence, I:59-96, 1987.

18. C. Sierra, J. Sabater, J. Augusti, and P. Garcia. SADDE: Social agents design driven by equations. In F. Bergenti, M. Gleizes, and F. Zambonelli, editors, Methodologies and software engineering for agent systems. Kluwer Academic Publishers, 2004

19. M. Sims, D. Corkill, and V. Lesser. Separating Domain and Coordination in Multi-Agent Organizational Design and Instantiation. In Proceedings of the International Conference on Intelligent Agent Technology (IAT 2004), Beijing, China, September 2004.

20. M. Sims, C. Goldman, and V. Lesser. Self-Organization through Bottom-up Coalition Formation. In Proceedings of Second International Joint Conference on Autonomous Agents and MultiAgent Systems (AAMAS 2003), pages 867-874, Melbourne, AUS, July 2003. ACM Press.

21. R. G. Smith. The contract net protocol: High-level communication and control in a distributed problem solver. IEEE Transctions on Computers, 29(12):1104-1113, 1980.

22. K. Sycara, K. Decker, and M. Williamson. Middleagents for the internet. In Proceedings of IJCAI-97, January 1997.

23. M. Tambe. Towards flexible teamwork. Journal of Artificial Intelligence Research, 7:83-124, 1997.

24. M. Tambe, J. Adibi, Y. Alonaizon, A. Erdem, G. A. Kaminka, S. Marsella, and I. Muslea. Building agent teams using an explicit teamwork model and learning. Artificial Intelligence, 110(2):215-239, 1999.

25. R. M. Turner. The tragedy of the commons and distributed AI systems. In Proceedings of the 12 th International Workshop on Distributed Artificial Intelligence, pages $379-390$, Hidden Valley, Pennsylvania, 1993. 